\title{
Panorama da prótese total no Brasil: um estudo bibliométrico
}

Overview of total prosthesis in Brazil: a bibliometric study

Panorama de prótesis total en Brasil: un estudio bibliométrico

\author{
Lays Mayara Macena SANTOS ${ }^{1}$ \\ José André Carneiro da SILVA ${ }^{1}$ \\ Cristine D'Almeida BORGES ${ }^{2}$ \\ Ana Regina Oliveira MOREIRA ${ }^{2}$ \\ Leopoldo Cosme SILVA ${ }^{2}$ \\ Valdeci Elias dos SANTOS JÚNIOR ${ }^{2}$ \\ Marília de Lima SOARES ${ }^{3}$ \\ Priscylla Gonçalves Correia Leite de MARCELOS \\ Diego Moura SOARES ${ }^{4}$
}

${ }^{1}$ Discente da Universidade Osman da Costa Lins (UNIFACOL), 55612- 285 Vitória de Santo Antão - PE, Brasil

${ }^{2}$ Professor Adjunto da Universidade Federal de Alagoas (UFAL), 57072-900 Macei - AL, Brasil

${ }^{3}$ Professor da Faculdade de Boa Viagem (FBV), 51200-060 Recife - PE, Brasil

${ }^{4}$ Professor Adjunto da Faculdade de Integração do Sertão (FIS) 56909-205 Serra Talhada - PE, Brasil

Tutor e Coordenador dos Laboratórios de Odontologia da Faculdade Pernambucana de Saúde (FPS) 51150-000 Recife - PE, Brasil

\section{Resumo}

Objetivo: O presente trabalho objetiva avaliar a produção científica publicadas nos anais do Congresso da Sociedade Brasileira de Pesquisa Odontológica ( $\mathrm{SBPqO}$ ) acerca da prótese total convencional. Metodologia: Foi realizado um estudo observacional retrospectivo, a partir da observação indireta e a análise de dados secundários, baseado nos trabalhos apresentados na Reunião Anual da SBPqO no período de 2014 a 2018. Foram analisados as variáveis nível de satisfação e qualidade de vida; deslocamento e inclinação; vínculo ao serviço público; infecção e contaminação; técnicas de moldagens; higiene; planejamento; estabilidade e retenção; e, materiais utilizados. Além disso, foram coletadas informações das subdivisões de regiões e estados, com o objetivo de verificar dentro da prótese total o panorama no Brasil no intervalo de cinco anos. Os dados foram catalogados e analisados com o auxílio do software SPSS na versão 16, e apresentados por meio da estatística descritiva. Resultados: Os resultados encontrados mostram que as Regiões Sudeste e Nordeste representam 56,9\% e 24,7\% da produção científica nacional sobre prótese respectivamente; $79,57 \%$ dos trabalhos foram realizados em instituições públicas; os temas mais pesquisados sobre prótese total é o nível de satisfação dos usuários e estabilidade da prótese; o número de publicações é muito flutuante ao longo dos anos. Conclusão: Conclui-se que existem muitas lacunas a serem abordadas sobre próteses totais, como os materiais disponíveis, planejamento e técnicas de moldagem.

Descritores: Prostodontia; Bibliometria; Prótese Total; Prótese Dentária.

\section{Abstract}

Objective: This present paper aims to evaluate the scientific production published in the annals of the Congress of the Brazilian Society of Dental Research (SBPqO) about the conventional full denture. Methodology: A retrospective observational study was carried out, based on indirect observation and the analysis of secondary data, based on the works presented at the Annual Meeting of the Brazilian Society for Dental Research (SBPqO) in the period from 2014 to 2018. The variables level of satisfaction and quality of life; displacement and inclination; link to the public service; infection and contamination; molding techniques; hygiene; planning; stability and retention; and, materials used. In addition, information was collected from the subdivisions of regions and states, with the aim of verifying the panorama in Brazil in the five-year interval within the total prosthesis. The data were cataloged and analyzed using the SPSS software in version 16, and presented using descriptive statistics. Results: The results found show that the Southeast and Northeast Regions represent 56.9\% and 24.7\% of the national scientific production on prosthesis; $79.57 \%$ of the works were carried out in public institutions; the most researched topics on total prosthesis are the level of user satisfaction and stability of the prosthesis; the number of publications is very fluctuating over the years. Conclusion: It is concluded that there are many gaps to be addressed about complete dentures, such as the materials available, planning and molding techniques

Descriptors: Prosthodontics; Bibliometrics; Denture, Complete; Dental Prosthesis.

\section{Resumen}

Objetivo: El presente trabajo tiene como objetivo evaluar la producción científica publicada en los anales del Congreso de la Sociedad Brasileña de Investigación Dental ( $\mathrm{SBPqO}$ ) sobre la prótesis total convencional. Metodología: Se realizó un estudio observacional retrospectivo, basado en la observación indirecta y el análisis de datos secundarios, en base a los trabajos presentados en la Reunión Anual SBPqO de 2014 a 2018. Se analizaron las variables nivel de satisfacción y calidad de vida; desplazamiento e inclinación; enlace al servicio público; infección y contaminación; técnicas de moldeo; higiene; planificación; estabilidad y retención; y, materiales utilizados. Además, se recopiló información de las subdivisiones de regiones y estados, con el objetivo de verificar el panorama en Brasil dentro de los cinco años de la prótesis total. Los datos se catalogaron y analizaron utilizando el software SPSS en la versión 16, y se presentaron utilizando estadísticas descriptivas. Resultados: Los resultados encontrados muestran que las regiones sudeste y noreste representan el $56.9 \%$ y el $24.7 \%$ de la producción científica nacional sobre prótesis, respectivamente; El 79,57\% de las obras se realizaron en instituciones públicas; Los temas más investigados sobre prótesis total son el nivel de satisfacción del usuario y la estabilidad de la prótesis; El número de publicaciones es muy fluctuante a lo largo de los años. Conclusión: se concluye que hay muchas lagunas que se deben abordar sobre las dentaduras postizas completas, como los materiales disponibles, las técnicas de planificación y moldeo.

Descriptores: Prostodoncia; Bibliometría; Dentadura Completa; Prótesis Dental.

\section{INTRODUÇÃO}

O edentulismo é caracterizado pela perda dos dentes, parcial e/ou total, podendo ser congênito ou ter sido obtido durante a vida. A ausência congênita é a forma mais grave por perdurar por toda vida dos indivíduos que se encontram em condições precárias, meios sociais, deficiência no serviço público oferecido, patologias e traumatismos ${ }^{1}$. Alterações na fala, mastigação, fonação, respiração e ATM
(Articulação Temporomandibular) podem ser fala, mastigação, fonação, respiração e ATM (Articulação Temporomandibular) podem ser desencadeadas pela ausência dos dentes, além de comprometer a estética e autoestima. Dessa forma o uso das próteses dentárias é de suma importância para tratar problemas fisiológicos, físicos e psicossocial do indivíduo ${ }^{2}$. 
Entretanto, por muito tempo a odontologia era conhecida por ser mutiladora, curativa e de um alto custo, sem atentar para o aspecto reabilitador ${ }^{1}$. Nesse contexto, a prótese dentária tem como propósito recuperar a estética e as funções do sistema estomatognático que estar ausente pela perda dos elementos dentários. Entretanto, para uma reabilitação adequada e eficiente é necessário analisar cada caso, para planejar e traçar um tratamento eficiente e adequado as expectativas do paciente ${ }^{3}$.

Para a Organização Mundial de Saúde (OMS), uma de suas metas globais para o ano de 2000, era de que pelo menos 50\% dos indivíduos com idades entre $65-74$ anos, tivessem pelo menos 20 dentes. De acordo com a Pesquisa Nacional de Saúde Bucal em seu estudo "SB Brasil" realizado em 2010, apenas $11,5 \%$ dos brasileiros com essa faixa etária enquadravam-se nas metas proposta pela OMS. Contudo, é sabido que apesar de ocorrer uma redução das perdas dentárias nos indivíduos mais jovens, o mesmo não ocorre na população idosa ${ }^{4}$.

Em consonância ao estabelecimento da Equipe de Saúde da Bucal (ESB), em 2001, e da Política Nacional de Saúde Bucal (PNSB), em 2004, verifica-se que o índice CPO-d do Brasil apresenta melhoras nas últimas décadas, revelando projeções de declínio do edentulismo entre adolescentes, adultos jovens e de meia-idade ${ }^{5,6}$. Ainda assim, o edentulismo é um fenômeno complexo, envolvendo multiplos fatores a serem controlados, tais com a incapacidade do Sistema Único de Saúde (SUS) de atender a demanda da população, inviabilidade financeira da população de custeio de tratamentos dentários, e o despreparo dos profissionais, que executam multilações em detrimento de técnicas conservadoras, acabando por incorrer a extração dentária 5 .

Em âmbito nacional, o cirurgião-dentista manifesta significativo valor no que se refere a acessibilidade da população de forma gratuita e universal. O Ministério da Saúde em 2005 implantou os centros de especialidades Odontológicas (CEO) que servem de suporte para os centros de saúde famíliar, direcionando os serviços de endodontia, periodontia, lesões bucais, cirurgia oral menor e prótese, nesse ultimo caso, por meio dos Laboratórios Regionais de Próteses Dentárias (LRPD), já que o Brasil tem um número elevado de indivíduos edêntulos?

Entender o nível de satisfação dos pacientes e reconhecer o cenário literário no estudo e planejamento da prótese faz-se necessário para buscar melhorar o nível de atendimento e da qualidade nas reabilitações. Vários tipos de estudos podem ser usados como base para reconhecer esse cenário, como, por exemplo, os estudos bibliométricos. Esse tipo de estudo contempla análises estatísticas nos padrões que aparecem nas publicações e uso dos documentos. Essa abordagem auxilia na compreensão de novas demandas e futuras tendências de pesquisas ${ }^{4,8}$.

Diante do exposto, apesar da quantidade de informações disponíveis relativas ao uso de próteses dentárias, pouco se tem documentado sobre planejamento, confecção, e análise da satisfação do usuário de prótese total no país. Portanto, o presente estudo tem como objetivo avaliar a produção científica publicadas nos anais do Congresso da Sociedade Brasileira de Pesquisa Odontológica (SBPqO) acerca da prótese total convencional. Além disso, traz um panorama situacional das pesquisas sobre próteses convencionais para estudantes e profissionais da odontologia, através de um estudo bibliométrico.

\section{MATERIAL E MÉTODO}

- Delineamento do estudo

Foi realizado estudo observacional retrospectivo, a partir da observação indireta e a análise de dados secundários, baseado nos trabalhos apresentados na Reunião Anual da $\mathrm{SBPqO}$ no período de 2014 a 2019. A escolha pelos anais do SBPqO foi motivada pela relevância que a Reunião Anual apresenta para o desenvolvimento da pesquisa científica no país, já que o encontro reune trabalhos desenvolvidos por acadêmicos, pós-graduandos, professores e pesquisadores. Além disso, todos os dados utilizados neste estudo são de acesso público por toda comunidade científica. Dessa forma, é possível obter um panorama de tudo que vem sendo feito pela comunidade científica a respeito do tema 9. Foram examinados 188 resumos publicados nos anais das Reuniões da SBPqO no período de 2014 a 2018, e desse conjunto, 106 resumos tratavam sobre prótese total e os aspectos pretendidos nessa pesquisa. Esses 106 trabalhos selecionados para constituir a amostragem dessa pesquisa foram publicados no intervalo de 2014 a 2018. Nestes trabalhos foram analisados as variáveis nível de satisfação e qualidade de vida, deslocamento e inclinação, vínculo ao serviço público, infecção e contaminação, técnicas de moldagens, higiene, planejamento, estabilidade/ retenção e materiais utilizados. Além disso, foram coletadas informações das subdivisões de regiões e estados, com o objetivo de verificar dentro da prótese total o panorama no Brasil no intervalo de cinco anos. Outros dados coletados foram relativos à instituição onde o trabalho foi desenvolvido, se a instituição é privada, Estadual ou Federal, e, por fim, o tipo do trabalho (se revisão de sistemática), relato de caso clínico ou pesquisa.

\section{- Coleta de dados}

A seleção se deu a partir da leitura na íntegra de todos os resumos referente a Prótese Total Convencional, por dois pesquisadores devidamente calibrados. Os resumos que suscitaram dúvidas pelos 
dois avaliadores durante a fase de triagem foram mantidos para uma avaliação mais detalhada por um terceiro examinador. Os critérios de exclusão aplicados foram trabalhos de revisão de literatura. Como critérios de inclusão foram: 1) trabalhos clínicos, pesquisa e de revisão sistemática que observaram a prótese total convencional e sobre implante em todos os seus aspectos, bem como 2) trabalhos científicos aprovados de todas as categorias dos anais foram avaliadas. Na primeira fase da busca, com a aplicação do descritor em saúde "prótese total", foram identificados 188 trabalhos. A partir disso, os resumos tiveram os títulos e corpo de texto examinados, observando-se os critérios de elegibilidade. Nesse ponto, 82 destes trabalhos foram excluídos da pesquisa por não se encaixarem nos critérios, sendo 46 destes por tratarem de prótese total sobre implante, e 36 por se desviar dos objetos de análise pretendidos nesse estudo. Por fim, 106 trabalhos restantes foram escolhidos para análise qualitativa (Figura 1).

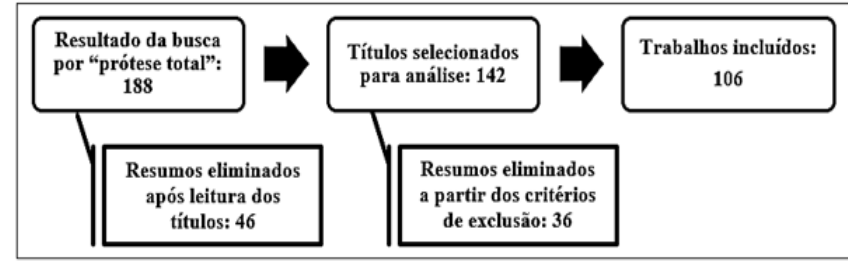

Figura 1: Esquema representativo do processo de busca pelo descritor "prótese total".

\section{- Análise de dados}

Para a mensuração dos resultados foi desenvolvida uma planilha própria, no programa Excel 2013, versão Professional Plus. Os dados coletados foram baseados em estudos publicados anteriormente por nossa equipe que utilizaram metodologias semelhantes $^{9-12}$. Os dados foram catalogados e analisados com o auxílio do software SPSS na versão 16, e apresentados por meio da estatística descritiva (frequências absoluta e percentual).

\section{RESULTADOS}

Conforme demonstrado no Gráfico 1, um total de 19 resumos foram publicados em 2014, 36 resumos em 2015, 27 trabalhos no ano de 2016, 13 trabalhos no ano de 2017 e por fim 11 resumos publicados no ano de 2018, totalizando 106 estudos. Nos trabalhos incluídos, verificou-se o desempenho em todas as regiões do país (Tabela 1).

Entretanto, 13 trabalhos não mencionam a localidade onde o estudo foi executado. Verifica-se uma predominância das regiões Sudeste e Nordeste no desenvolvimento de pesquisas relacionadas a prótese total. A região Sudeste representa $56,9 \%$ da produção científica do universo dessa pesquisa, ou seja, 53 resumos publicados. O Nordeste por sua vez é a segunda região com maior número de publicações, responsável por 23 trabalhos ou $24,7 \%$ dos trabalhos apresentados (Tabela 1).
No tocante as pesquisas contempladas, 93 trabalhos registraram o local de execução do trabalho. Entre esses trabalhos, 79,57\% das pesquisas foram desenvolvidas em instituições públicas, enquanto apenas $20,43 \%$ dos estudos foram desenvolvidos em instituições particulares (Tabela 2). Dos temas estudados, observa-se uma predominância do assunto nível de satisfação do paciente (30), seguido de estudos que avaliam a estabilidade da prótese (22).

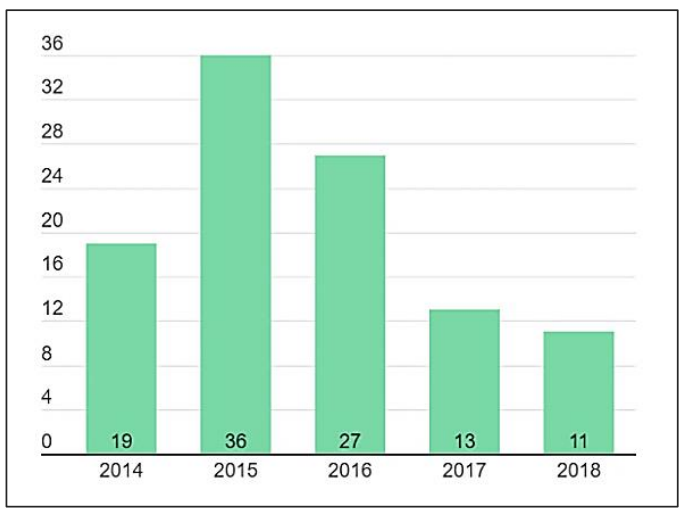

Gráfico 1: Gráfico de distribuição dos trabalhos por ano.

Tabela 1. Distribuição dos trabalhos de acordo com a região brasileira

\begin{tabular}{lcc}
\hline Região / Estado & Frequência (no / \%) \\
\hline Centro-Oeste & 7 & $7,55 \%$ \\
Nordeste & 23 & $24,7 \%$ \\
Norte & 1 & $1,09 \%$ \\
Sudeste & 53 & $56,9 \%$ \\
Sul & 9 & $9,76 \%$ \\
\hline Total & $\mathbf{9 3}$ & $\mathbf{1 0 0 \%}$ \\
\hline Teste Qui-Quadrado & &
\end{tabular}

Tabela 2. Distribuição dos trabalhos de acordo com o tipo de instituição

\begin{tabular}{|c|c|c|}
\hline Instituição & \multicolumn{2}{|c|}{ Frequência( $\left(n^{0} / \%\right)^{*}$} \\
\hline PÚBLICA & 74 & $79,57 \%$ \\
\hline Centro-Oeste & 6 & $6,42 \%$ \\
\hline Nordeste & 18 & $19,35 \%$ \\
\hline Norte & 1 & $1,09 \%$ \\
\hline Sudeste & 42 & $45,16 \%$ \\
\hline Sul & 7 & $7,55 \%$ \\
\hline PRIVADA & 19 & $20,43 \%$ \\
\hline Centro-Oeste & 1 & $1,09 \%$ \\
\hline Nordeste & 5 & $5,35 \%$ \\
\hline Norte & 0 & $0 \%$ \\
\hline Sudeste & 11 & $11,81 \%$ \\
\hline Sul & 2 & $2,18 \%$ \\
\hline TOTAL & 93 & $100 \%$ \\
\hline
\end{tabular}

DISCUSSÃO

Estudos cientométricos revelam que a produção científica odontológica brasileira tem evoluído ao longo dos anos, ocupando lugar de destaque no cenário científico internacional. De forma geral, embora a produção científica total é considerada de baixo impacto, a literatura odontológica é responsável por $7 \%$ de toda produção científica nacional $^{13}$. O conteúdo das publicações da $\mathrm{SBPqO}$ são de grande relevância e tradição para comunidade odontológica, pois este órgão representa 
a subdivisão brasileira da Internacional Association for Dental Research, o qual constitui o órgão máximo de pesquisa odontológica mundial ${ }^{14}$. Consoante a isso, esse estudo observou o panorama e o desenvolvimento da produção científica, através da análise focalizada nas publicações dos anais da reunião anual da $\mathrm{SBPqO}$.

Nesse contexto, o estudo bibliométrico serve para compreender a atividade científica e o interesse da comunidade odontológica por áreas específicas ${ }^{15}$. Conforme o levantamento de dados dessa pesquisa, 106 trabalhos tratam de prótese total e suas especificidades, valor relativamente baixo ou assimétrico com a produtividade total, vide o amplo número de trabalhos apresentados anualmente neste congresso. É importante destacar que as pesquisas científicas são igualmente relevantes à práxis clínica atual quanto os livros e manuais, pois esta valoriza a prática da Odontologia Baseada em Evidências ${ }^{16}$. Logo, o engajamento no estudo das próteses dentárias é um importante recurso à reabilitação e manutenção da saúde humana, visto que está diretamente relacionado a problemas de dor, mastigação, fonação, relacionamento social dos edêtulos e outros gravos como a desnutrição ${ }^{17}$.

Quanto ao volume de publicações ao longo dos anos, a mensuração dos estudos encontrados apresenta oscilação ao longo dos anos, revelando um desvio padrão de cerca de 9,26 pontos de diferença, sendo o ano de 2015 aquele com maior número de publicações. Ao ser avaliado a tendência das especialidades e assuntos das pesquisas constata-se um direcionamento destas em sentido concordante com a demanda de casos que chegam aos centros de atendimento odontológico do SUS e nas clínicasescola das universidades ${ }^{18}$. Em consonância a esta afirmativa Celeste e Warmling ${ }^{19}$ destacam que as variações das temáticas representam as alterações no cenário epidemiológico local, nas políticas públicas em saúde, e principalmente nas evoluções tecnológicas do setor.

No que se refere a incidência de trabalhos por região do país, os dados destacam que o Sudeste é responsável por 56,9\% dos trabalhos publicados, seguido do Nordeste $(24,7 \%)$. Fato este que pode ser explicado pela existencia de um parque científicotecnológico no país em torno de São Paulo ${ }^{20}$, pela maior concentração de cursos de graduação e pósgraduação, bem como maior densidade de investimento pelo governo e órgãos de fomento nos estados da região Sudeste ${ }^{15}$. Pode-se afirma tambem que os demais programas de pós-graduação espalhados pelo país são mais recentes e por isso ainda não alcançaram a mesma conceituação dos programas da região Sudeste ${ }^{21}$.

Em linhas gerais, o financiamento das pesquisas em saúde se dá principalmente por meio dos órgãos públicos como o Conselho Nacional de
Desenvolvimento Científico e Tecnológico (CNPq), a Coordenação de Aperfeiçoamento de Pessoal de Nível Superior (CAPES) e as agências de fomento regionais ${ }^{22}$. Em concordancia a esta informação os resultados desta investigação constatou a frequência de $79,57 \%$ do desenvolvimento de trabalhos nas instituições públicas de ensino superior, destacando mais uma vez as regiões Sudeste e Nordeste. Considerando que o incentivo a realização de pesquisa é essencial à prática clínica e manutenção da produtividade científica em saúde, foi criada a Agenda Nacional de Prioridades de Pesquisa em Saúde (ANPPS), com o propósito de alinhar as prioridades da pesquisa nacional com as prioridades do SUS ${ }^{13}$.

Referente às variáveis coletadas pelos pesquisa, os principais temas trazido nos resumos sobre prótese total foram estabilidade e o nível de satisfação dos usuários. O quesito estabilidade está intimamente ligado à satisfação do usuário e torna-se importante, pois a instalação deve estar perfeitamente ajustada ao tecido de suporte, a fim de alcançar boa retenção, promovendo estabilidade e conforto ao paciente $^{23}$ evitando lesões bucais, prejuízo estético e funcional $^{24}$. O também reestabelicimento da estética influencia de sobremaneira a satisfação do usário das proteses ${ }^{25}$.

Um aspecto muito relevante à satisfação geral do paciente reabilitado por prótese total é a longevidade das próteses, a qual está intimamente ligada à sua correta higienização e manutenção, tanto quanto sua anatomia e material ${ }^{26}$. Contudo, apenas 9 trabalhos relacionaram a higiene, e 9 (n) a pigmentação associada a contaminação e o consumo de alimentos.

Ao $\log$ o do período avaliado, 1 trabalho apenas avaliou o tipo de material utilizado na estrutura da prótese total e 3 versaram sobre métodos de planejamento. Historicamente, vários e diferentes materiais foram utilizados para moldagem e estrutura das próteses totais até chegar a tecnologia atual, de forma a agradar funcional e esteticamente ao paciente $^{27}$. Segundo Barbosa e colaboradores ${ }^{25}$, é possível predizer o sucesso da reabilitação por prótese quando é feito um excelente planejamento do tratamento, e por isso a importância do seu estudo por parte dos cirurgiões-dentistas.

Por fim, a técnica de bibliometria aplicada nessa pesquisa permite avaliar os viéses do interesse da comunidade odontológica e a disseminação de novas informações científicas sobre prótese total. Para tanto, é necessário que o cirurgião-dentista empregue uma visão crítica não só sobre a atualização das evidências apresentadas, as quais podem ser utilizadas na prática clínica, como também analisar o curso da ciência e identificar as lacunas da literatura atual ${ }^{28}$. 


\section{CONCLUSÃO}

Seguindo a tendência mundial de avaliar o panorama e a expansão da pesquisa científica, esse estudo representa um bom recurso avaliativo do empenho da comunidade odontológica em estudar a prótese total. Baseado nos resultados obtidos, o principal foco da pesquisa acerca de prótese total no país é sobre a estabilidade da prótese e o nível de satisfação dos usuários, como ponto de partida para estudo dos fatores relacionados. E por fim, pode-se concluir que o desenvolvimento da produção científica odontológica é diretamente proporcional à disponibilidade de financiamento à pesquisas no país, visto o centro de promoção das pesquisas como sendo os programas públicos de ensino, com epicentro no parque científico-tecnológico da região Sudeste. Finalmente, esse trabalho serve como base para pesquisas posteriores com o objetivo de desenvolver a pesquisa nessa área.

\section{REFERÊNCIAS}

1. Melo M, Borges RN, Freitas GC, Prado MM, Melo TG, Melo TCAS. Verificação do uso de próteses dentárias em servidores da UFG. Robrac. 2015;24(69):92-4.

2. Evangelista Souza S, Valadares Oliveira L, Pinheiro Freitas A, Andrade Meyer G, Carneiro Leão Gabarto M. Perfil sócio-econômico de pacientes desdentados totais reabilitados na Faculdade de Odontologia da Universidade Federal da Bahia, Brasil. Rev cubana estomatol. 2015;52(1).

3. Nóbrega DRM, Lucena AG, Medeiros LADM, Farias TSS, Meira KRS, Mahon SMOD. Avaliação da utilização e hábitos de higiene em usuários de prótese dentária removível. Rev Bras Odontol. 2016;73(3):193-97.

4. Azevedo JS, Azevedo MS, Oliveira LJC, Correa MB, Demarco FF. Uso e necessidade de prótese dentária em idosos brasileiros segundo a Pesquisa Nacional de Saúde Bucal (SBBrasil 2010): prevalências e fatores associados. Cad Saúde Pública. 2017;33(8): e00054016.

5. Cardoso M, Balducci I, Telles DM, Lourenço EJV, Nogueira Junior, L. Edentulism in brazil: trends, projections and expectations until 2040. Cienc saúde coletiva. 2016; 21(4):1239-46.

6. Silva ET, Oliveira RT, Leles CR. O edentulismo no brasil: epidemiologia, rede assistencial e produção de próteses pelo sistema único de saúde. Tempus. 2015;9(3):121-34.

7. Silva FR, Padilha EZ, Baldani MH. Serviços odontológicos especializados nas cidades médias não metropolitanas do estado do Paraná, entre 2003 e 2010: estudo exploratório. Epidemiol Serv Saúde. 2013;22(4):641-50.

8. Pereira VR, Carvalho MM, Rotondaro RG. Um estudo bibliométrico sobre a evolução da pesquisa da qualidade em serviço. Production. 2013; 23(2):312-28

9. Maciel MMSA, Silva KBN, Melo JGA, Soares DM. Metodologia ativa aplicada ao ensino odontológico: um panorama nacional a partir de um estudo bibliométrico. Arch Health Invest. 2019;8(2):74-8.

10. Melo JGA, Soares DM. Análise bibliométrica do uso de células-tronco em pesquisas odontológicas. Arch Health Invest. 2019;8(12):766-70.

11. Soares D, Maciel M, Figueredo-Filho A, Melo J. Brazilian scientific production in periodontics: a national panorama from a bibliometric study. Rev Clin Periodoncia Implantol Rehabil Oral. 2019; 12(2); 66-9.

12. Queiroz VKP, Soares DM. Pesquisas odontológicas relacionadas com microcefalia: um estudo bibliométrico. Arch Health Invest 2019;8(11):681-85.

13. Gomes D, Agnoletto IG, Souza ML, Spiger V, Jakymiu JRG, Fugii EC et al. A produção científica da Odontologia e a Agenda Nacional de Prioridades de Pesquisa em Saúde. Rev ABENO. 2017;17(2):11-21.

14. Primo BT, Grazziotin-Soares R, Bertuzzi D, Claudy MP, Hernandez PAG, Fontanella VRC. Produção científica da ULBRA: análise do número e do delineamento das pesquisas publicadas nos suplementos da Brazilian Oral Research (SBPqO). Stomatos. 2010; 16(31):69-76.

15. Souza JGS, Popoff DAV, Oliveira RCN, Almeida ER, Martelli Junior H, Martins AMEBL. Profile and scientific production of Brazilian researchers in dentistry. Arq Odontol. 2016;52(1):13-22.

16. Navarro P, Chuhuaicura P, Soto-Faúndez N, Soto C. Diseños de investigación y pruebas estadísticas utilizadas en revistas odontológicas de la red SciELO. Av Odontoestomatol. 2019; 35(1):19-25.

17. Aguiar VR, Celeste RK. Necessidade e alocação de laboratórios regionais de prótese dentária no brasil: um estudo exploratório. Ciênc saúde coletiva. 2015;20:3121-28.

18. Dias AA, Narvai PC, Rêgo DM. Tendências da produção científica em odontologia no Brasil. Rev Panam Salud Publica. 2008;24(1):54-60.

19. Celeste RK, Warmling CM. Produção bibliográfica brasileira da Saúde Bucal Coletiva em periódicos da saúde coletiva e da Odontologia. Ciênc saúde coletiva. 2014;19(6):1921-32.

20. Cavalcanti AL, Melo TRNB, Barroso KMA, Souza FECD, Maia AMA, Silva ALDO. Perfil da pesquisa científica em Odontologia realizada no Brasil. Pesqui bras odontopediatria clin integr. 2004;4(2):99-104.

21. Gonçalves APR. Cenário atual e inserção internacional da pesquisa Odontológica brasileira [tese]. Pelotas: Universidade Federal de Pelotas; 2019.

22. Gabardo MCL, Copelli FA, Tuzzi AL, Trentin G, 
Lima J, Tomazinho FSF, et al. Pesquisa científica em Endodontia apresentada na Reunião Anual da Sociedade Brasileira de Pesquisa Odontológica: análise bibliométrica de 2010 a 2018. Rev ABENO. 2019;19(3):144-52.

23. Barbosa D, Barão VAR, Assunção WG, Gennari Filho H, Goiato MC. Instalação de prótese total: uma revisão. Rev Odontol UNESP. 2006; 35(1):53-60.

24. Abreu CW, Munhoz E. Os fatores que influenciam na satisfação do paciente submetido a tratamento de prótese total convencional. Hu Rev. 2011;37(4):413-19.

25. Martins LMJ. Vantagens e desvantagens da prótese total acrílica e a prótese total sobre implante: uma revisão narrativa da literatura [disseetação]. Portugal: Instituto Universitário de Ciências da Saúde; 2018.

26. Marchini L, Montenegro FLB, Cunha VDPP, Santos JFF. Prótese dentária na terceira idade: considerações clínicas e preventivas diversas. Rev Longeviver. 2010;1.

27. Grings JS. Protocolo de branemark: uma revisão de literatura [monografia]. Porto Alegre: Faculdade de Odontologia da Universidade Federal do Rio Grande do Sul; 2018

28. Gonzalez Ramos RM, Reyes SAR, Grandal OV, Padrón ER, Pedroso LH. Caracterización bibliométrica de la producción científica de la Facultad de Estomatología "Raúl González Sánchez", 2011-2015. Rev cubana estomatol. 2018;55(2):8-14.

\section{CONFLITO DE INTERESSES}

Os autores declaram não haver conflitos de interesse.

\section{AUTOR PARA CORRESPONDÊNCIA}

Priscylla Gonçalves Correia Leite de Marcelos

Av. Lourival de Melo Mota, s/n, Tabuleiro do Martins, 57072-900 Maceió-AL, Brasil

Email: priscylla.marcelos@ foufal.ufal.br

Submetido em 27/06/2020

Aceito em 15/12/2020 